\title{
Research Paper: Functional Brain Response to Emotional Musical Stimuli in Depression, Using INLA Approach for Approximate Bayesian Inference
}

Parisa Naseri ${ }^{1}$ (D), Hamid Alavi Majd ${ }^{1^{*}}$ (D), Seyyed Mohammad Tabatabaei² (D), Naghmeh Khadembashi ${ }^{3}$ (D), Seyed Morteza Najibi ${ }^{4}$, Atiye Nazari

1. Department of Biostatistics, School of Paramedical Sciences, Shahid Beheshti University of Medical Sciences, Tehran, Iran.

2. Department of Medical Informatics, School of Medicine, Mashhad University of Medical Sciences, Mashhad, Iran.

3. Department of English Language, School of Allied Medical Sciences, Shahid Beheshti University of Medical Sciences, Tehran, Iran

4. Department of Statistics, School of Sciences, Shiraz University, Shiraz, Iran.

\begin{tabular}{|l|l|}
\hline $\begin{array}{l}\text { Use your device to scan } \\
\text { and read the article online }\end{array}$ & $\begin{array}{l}\text { Crtation: Naseri, P., Alavi Majd, H., Tabatabaei, S. M., Khadembashi, N., Najibi, S. M., \& Nazari, A. (2021). Functional Brain } \\
\text { Response to Emotional Musical Stimuli in Depression, Using INLA Approach for Approximate Bayesian Inference. Basic and } \\
\text { Clinical Neuroscience, 12(1), 95-104. http://dx.doi.org/10.32598/bcn.9.10.480 }\end{array}$ \\
\hline dol'http://dx.doi.org/10.32598/bcn.9.10.480
\end{tabular}

\section{(i) (3)}

Article info:

Received: 24 Jun 2018

First Revision: 10 Jul 2018

Accepted: 10 Mar 2019

Available Online: 01 Jan 2021

Keywords:

Bayesian GLM approach, Unweighted graph-Laplacian, Integrated nested Laplace approximation, functional magnetic resonance imaging, Anterior cingulate cortex, Depression
A B S T RA C T

Introduction: One of the vital skills which has an impact on emotional health and well-being is the regulation of emotions. In recent years, the neural basis of this process has been considered widely. One of the powerful tools for eliciting and regulating emotion is music. The Anterior Cingulate Cortex (ACC) is part of the emotional neural circuitry involved in Major Depressive Disorder (MDD). The current study uses functional Magnetic Resonance Imaging (fMRI) to examine how neural processing of emotional musical auditory stimuli is changed within the ACC in depression. Statistical inference is conducted using a Bayesian Generalized Linear Model (GLM) approach with an Integrated Nested Laplace Approximation (INLA) algorithm.

Methods: A new proposed Bayesian approach was applied for assessing functional response to emotional musical auditory stimuli in a block design fMRI data with 105 scans of two healthy and depressed women. In this Bayesian approach, Unweighted Graph-Laplacian (UGL) prior was chosen for spatial dependency, and autoregressive (AR) (1) process was used for temporal correlation via pre-weighting residuals. Finally, the inference was conducted using the Integrated Nested Laplace Approximation (INLA) algorithm in the R-INLA package.

Results: The results revealed that positive music, as compared to negative music, elicits stronger activation within the ACC area in both healthy and depressed subjects. In comparing MDD and Never-Depressed (ND) individuals, a significant difference was found between MDD and ND groups in response to positive music vs negative music stimuli. The activations increase from baseline to positive stimuli and decrease from baseline to negative stimuli in ND subjects. Also, a significant decrease from baseline to positive stimuli was observed in MDD subjects, but there was no significant difference between baseline and negative stimuli.

Conclusion: Assessing the pattern of activations within ACC in a depressed individual may be useful in retraining the ACC and improving its function, and lead to more effective therapeutic interventions. 


\section{Highlights}

- The positive music, as compared to negative music, elicits stronger activation within the ACC area in both healthy and depressed subjects.

- A significant difference was found between MDD and ND groups in response to positive music vs negative music stimuli.

- The activations increase from baseline to positive stimuli and decrease from baseline to negative stimuli in ND subjects.

\section{Plain Language Summary}

Regarding the large amount of fMRI data and the spatiotemporal correlations, the computational cost of analysis is challenging. Because of the disability of the classical Generalized Linear Model (GLM) in considering fMRI data properties, some alternative Bayesian approaches have been proposed. A recently-developed Bayesian inference tool based on integrated Nested Laplace Approximation (INLA) has been employed in previous studies. This approach resulted in accurate results with reasonable computational time. INLA computes approximations to the posterior distributions and manage large data sets in a shorter time using the sparsity of Gaussian Markov Random Fields (GM- RFs). The present study uses INLA to identify areas reactive to positive and negative emotional stimuli between MDD and ND control participants. It is hypothesized that individuals with depression compared with ND control participants will show greater activations to negative stimuli and reduced responsiveness to positive stimuli in ACC.

\section{Introduction}

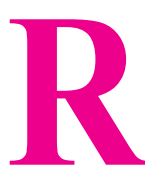

apid growth in functional Magnetic Resonance Imaging (fMRI) over the past two decades has led to considerable developments in our understanding of human brain mechanisms (Raichle, 2003). The objective of an fMRI study is to detect regional brain activity changes during the neural experiment. A vital skill is regulating emotions, impacting emotional health and well-being (Gross \& Muñoz, 1995). In recent years, the neural basis of this process has been studied extensively. Investigators use music as a powerful tool for eliciting and regulating emotions in clinical and laboratory research studies (Aselton, 2012; Clark, 1983; Clark \& Teasdale, 1985; Holzinger, Matschinger, \& Angermeyer, 2012; Lepping et al., 2016; Pignatiello, Camp, \& Rasar, 1986; Sloboda \& Juslin, 2001; Sutherland, Newman, \& Rachman, 1982; Västfjäll, 2001). One of the most common problems is depression, where about 121 million people worldwide suffer from this disorder. Depression leads to disability and is the second cause of the global disease burden by 2020 (Moussavi et al., 2007; Worlg Health Organization, 2000).

One of the ways of treating depression is music therapy. Music is a useful treatment that reduces stress and anxi- ety in depressed people (Aselton, 2012; Holzinger et al., 2012). Because of this significant therapeutic effect, music is a strong tool to impel mood, which is suitable for studying emotion regulation and is highly effective for mental diseases such as depression. So, more research is helpful to understand the function of emotional stimuli in treatments for depression to answer the question of why people with depressive symptoms choose music as a way to help themselves (Lepping et al., 2016).

In an fMRI study, each subject performed a series of tasks, and the changes in the Blood-Oxygen-Level-Dependent (BOLD) signal were measured during scans. Moreover, for each subject and at each voxel, more than hundreds of 3D volumes of BOLD measurements are collected (Daliri \& Behroozi, 2012; Lindquist, 2008; Nazari et al., 2019; Poldrack, Mumford, \& Nichols, 2011). The data have spatial and temporal correlations. Spatial correlation between thousands of voxels and temporal correlations at hundreds of time points at each voxel leads typically to massive amounts of highly complex data (Çelik, Dar, Yılmaz, Keleş, \& Çukur, 2019).

In addition to statistical modeling of fMRI data, which considers both spatial and temporal structures, the computational cost dealing with the analysis of such high dimensional data is challenging. Because of the disability of the classical Generalized Linear Model (GLM) in con- 
sidering fMRI data properties, some alternative Bayesian approaches have been proposed (Sidén, Eklund, Bolin, \& Villani, 2017; Teng, Nathoo, \& Johnson, 2017). In a Bayesian GLM, specific prior distributions are assumed for the task activation and other unknown parameters in the model. So, considering them with this likelihood, the Bayesian hierarchical model is composed.

Owing to a large amount of data, standard Markov Chain Monte Carlo (MCMC) methods are typically too time-consuming, so a recently-developed Bayesian inference tool based on integrated Nested Laplace Approximation (INLA) has been employed (Rue, Martino, \& Chopin, 2009). INLA method can compute approximations to the posterior distributions and manage large data sets in a shorter time using the sparsity of Gaussian Markov Random Fields (GMRFs). Moreover, INLA is faster than MCMC (Rue et al., 2017) and can be easily implemented using the R-INLA package (Mejia, Yue, Bolin, Lindren, \& Lindquist, 2020).

The present study uses fMRI data to identify areas reactive to positive and negative emotional stimuli between MDD and ND control participants. It is hypothesized that individuals with depression compared with ND control participants will show greater activations to negative stimuli and reduced responsiveness to positive stimuli in ACC. A Bayesian GLM approach is applied for statistical analysis, and inference is conducted using INLA.

\section{Materials and Methods}

\subsection{Subjects}

In this experiment, 19 participants with depression (MDD) and 20 never-depressed control individuals (ND) listened to emotional musical stimuli during scanning.

The Structured Clinical Interview for DSM Disorders, non-patient version (SCID-I/NP) was used to assess ND participants without a history of depression or other psychiatric disorders (First, 2002). Using the SCID-I/NP, the individuals in the MDD group experiencing a current depressive event at the time of scanning were determined.
For the present research, the brain images of subjects 11 and 1 from ND and MDD groups were selected, respectively. The chosen participants are women of the age of 29 years. The relevant data were obtained from the OpenfMRI database for the current analysis. Its accession number is ds000171 (Lepping et al., 2016).

\subsection{Stimuli}

Stimuli were presented in a block design paradigm (Figure 1) by E-Prime 2.0 software. During each functional run, the participants were exposed to 30-s blocks of positive, negative, and pure tone (baseline) samples. Emotionally evocative positive and negative musical examples from Western art music were used. Two repetitions of each block type were alternated between positive and negative stimuli. The baseline block between stimuli was repeated, and each run began and ended with a baseline block. Stimuli were presented through Magnetic Resonance (MR) compatible earbuds (Sensimetrics Corporation, Malden, MA) at $70 \mathrm{~dB}$, or as loud as comfortably possible to ensure the stimuli were heard over the noise of the scanner.

\subsection{Functional Magnetic Resonance Imaging}

In a single scanning session for each subject, an anatomical and 5 functional scanning runs were acquired. Scanning was conducted on a Siemens 3T Skyra scanner (Siemens, Erlangen, Germany). High-resolution T1-weighted anatomic images were acquired with a 3D MPRAGE sequence $(\mathrm{TR} / \mathrm{TE}=2300 / 2.01 \mathrm{~ms}$, flip angle $=9^{\circ}$, field of view $[\mathrm{FOV}]=256 \mathrm{~mm}$, matrix $=256 \mathrm{x}$ 192 , slice thickness $=1 \mathrm{~mm})$. Following structural scans, 5 gradient-echo BOLD sequences were acquired in 50 interleaved oblique axial slices at a $40^{\circ}$ angle (repetition time/ time to echo $[\mathrm{TR} / \mathrm{TE}]=3000 / 25 \mathrm{~ms}$, flip angle $=90^{\circ}$, field of view $[\mathrm{FOV}]=220 \mathrm{~mm}$, matrix $=64 \times 64$, slice thickness $=3 \mathrm{~mm}, 0 \mathrm{~mm}$ skip, in-plane resolution $=2.9 \mathrm{x}$ $2.9 \mathrm{~mm}, 105$ data points, $5 \mathrm{~min}: 24 \mathrm{~s}$ ). The first functional run of each subject was chosen for analysis.

\subsection{Data processing and statistical analysis}

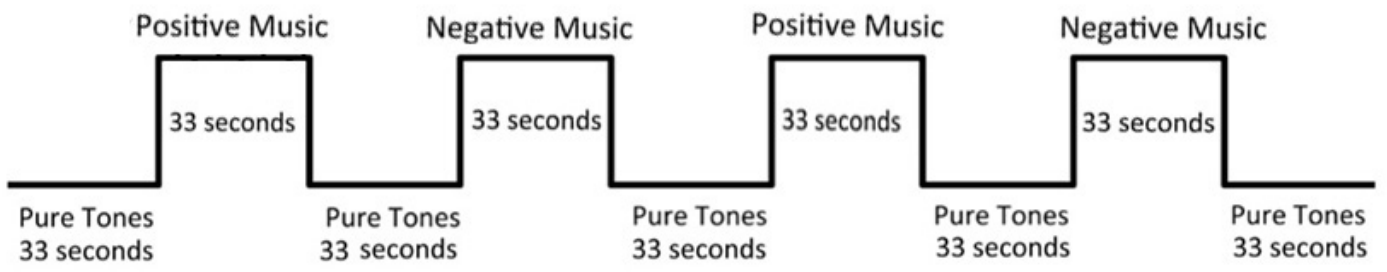

Figure 1. fMRI paradigm, functional run from the blocked emotional stimulus paradigm 


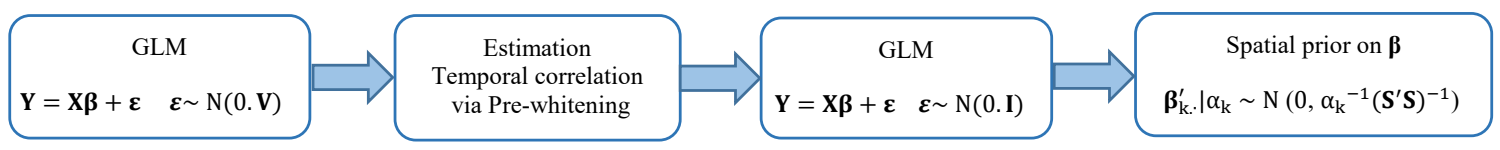

Figure 2. A schematic of the proposed model

NEUR SCIENCE

First, the pre-whitening approach is used to capture temporal correlation; Second: a spatial Bayesian hierarchical model is fitted to obtain the time series from the previous step.

The data were preprocessed with regard to the pipeline in Lepping et al (2016). and using the SPM12 software package (http:/www.fil.ion.ucl.ac.uk/spm/software/ spm12/), which includes removal of spatial distortions, motion realignment, distortion correction, alignment to the structural image, bias field correction and intensity normalization. Moreover, a Gaussian filter with $4 \mathrm{~mm}$ FWHM was used to smooth the images, and high-pass filtering was done with a cutoff point of $128 \mathrm{~s}$. These are standard steps in fMRI data preprocessing and must align the data into a common space and remove primary noise sources. The design matrix was generated by fitting a boxcar function to each time series convolved with a canonical hemodynamic response function.

\subsection{Statistical analysis}

Let $\mathrm{T}$ be the number of time points in the fMRI time series and $\mathrm{N}$ be the number of voxels in the brain. For a subject, the following GLM model is presented (Model 1) (Mejia et al., 2020):

$$
\text { (1) } y=\sum_{k=0}^{K} X_{k} \beta_{k}+\sum_{j=1}^{J} Z_{j} b_{j} \varepsilon \sim N(0 . V) \text {. }
$$

Here, $\mathrm{y}$ is a $\mathrm{TN} \times 1$ vector containing the $\mathrm{fMRI}$ time series of all voxels, and theandare $\mathrm{TN} \times \mathrm{N}$ design matrices for the activation amplitudes (including baseline) and nuisance signals, respectively. The matrix $\mathrm{V}$ is a $\mathrm{T} \times \mathrm{T}$ covariance matrix for an $\mathrm{AR}(\mathrm{p})$ process, where $\mathrm{p}$ is the degree of autoregressive.

The spatial correlation will enter via the Unweighted Graph-Laplacian (UGL) before each for $\mathrm{k}=0, \ldots, \mathrm{K}$, where $\mathrm{K}$ is the number of the task under investigation. UGL is defined as below (Model 2) (Sidén et al., 2017):

(2) $\beta_{k .}^{\prime} \mid \alpha \_k \sim N\left(0,\left(\alpha_{k} D_{\beta}\right)^{-1}\right)$,

where are a fixed spatial $\mathrm{N} \times \mathrm{N}$ precision matrix and $=$ are hyperparameters to be estimated from the data. There are several choices for but this study considers UGL, which has the number of adjacent voxels on the diagonal for each voxel and, on the condition that $i$ and $j$ are adjacent. When modeling each 2D slice separately, for voxels in the interior part of the brain, there exist 4's on the diagonal.
Generally, in the Bayesian approach, the full conditional distribution of each unknown parameter should be reached so that MCMC-based algorithms could be used to obtain samples from their posterior distributions and make Bayesian inferences using those samples. However, regarding fMRI data size and the complexity of the spatiotemporal model, the MCMC is slow in converge and is too time-consuming. As an alternative to MCMC, Rue et al. (2009) introduced a novel Bayesian computation tool based on Integrated Nested Laplace Approximations (INLA), which is implemented in the R-INLA package (Rue et al., 2009). For the first time, the INLA algorithm is used in the present study to inferences of the described model on volumetric fMRI data. It accurately approximates marginal posterior densities and computes all necessary estimates faster than MCMC techniques. For a more detailed explanation of the INLA method, refer to Blangiardo and Cameletti (2015).

To consider the temporal correlation of time series and reduce the computational cost of fitting the Bayesian model, the fMRI time courses were first pre-whitened by assuming an $\mathrm{AR}(\mathrm{p})$ process on the residuals from a classical GLM with uncorrelated errors. Here $p$ was considered as one (Bollmann, Puckett, Cunnington, \& Barth, 2018). Pre-whitened was done as follows (Monti, 2011):

(1) The number of $p$ autoregressive coefficients for each location in the brain was estimated.

(2) The pre-whitening matrix $\mathrm{W}$ for each location in the brain was computed, resulting in $\mathrm{N} \mathrm{T} \times \mathrm{T}$ matrices, where $\mathrm{N}$ is the number of voxels in the brain.

(3) Finally, the regression model in Equation (3) was fitted at each voxel to get estimates and standard errors for $\beta$ for each subject and voxel (Model 3).

(3) $W y=W X \beta+W \varepsilon, W \varepsilon \sim N(0 . I)$

A graphical illustration of the proposed model is shown in Figure 2. To account for noise due to subject motion, 6 rigid body realignment parameters estimated in the motion realignment stage of preprocessing were included in the model as nuisance covariates. Furthermore, linear 


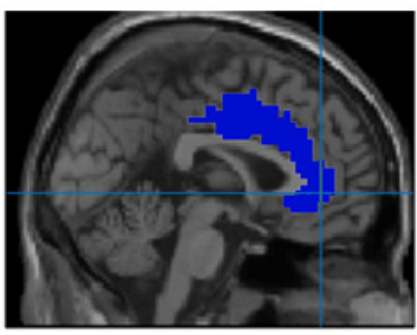

(a)

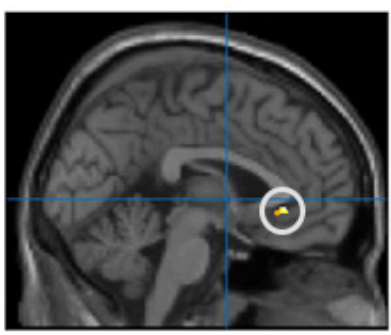

(b)
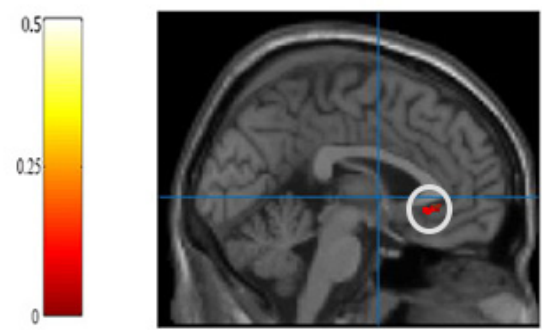

(c)

NEUR SCIENCE

Figure 3. Activation maps in response to music stimuli

A: The mask of ACC; B: Brain regions with stronger activation in response to positive music vs negative music for ND participant; $\mathrm{C}$ : Brain regions with stronger activation in response to positive music vs negative music for MDD participant.

and quadratic time terms were included for considering scanner drift.

A Region of Interest (ROI) analysis was conducted focusing on regions implicated in emotion processing in depression, including ACC (Brodmann areas [BA] 32 and 33, sgACC [BA25]).

The mask of the selected regions was made using the WFU PickAtlas toolbox in MATLAB R2016b software (Maldjian, Laurienti, Kraft, \& Burdette, 2003). All brain images were mapped on a standardized single T1 template in SPM (Oishi et al., 2009). The obtained data were prepared by programming in MATLAB R2016b software, and then model fitting was performed using the R-INLA package (http://www.r-inla.org).

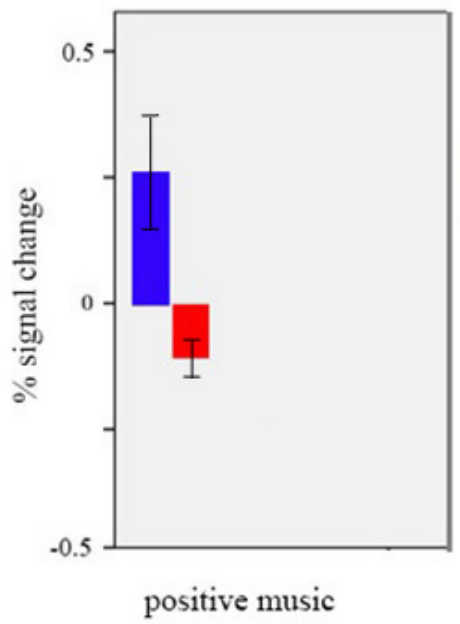

(a)

\section{Results}

After preprocessing the data, the ROI based analysis was conducted. The xjView program (http:/www.alivelearn.net/xjview) was applied for the illustration of brain activation maps.

The ACC region of the brain plays an essential and pivotal role in emotion formation, regulation, and processing. The reason to perform an ROI analysis is to avoid the difficulty faced in discerning the pattern of activity across conditions from an overall map. The other reason is to limit the testing to a region that is functionally defined based on interested functional regions.

After temporal estimation correlation via the prewhitening approach, the INLA method is applied to the

Figure 4. fMRI results group by positive-negative music

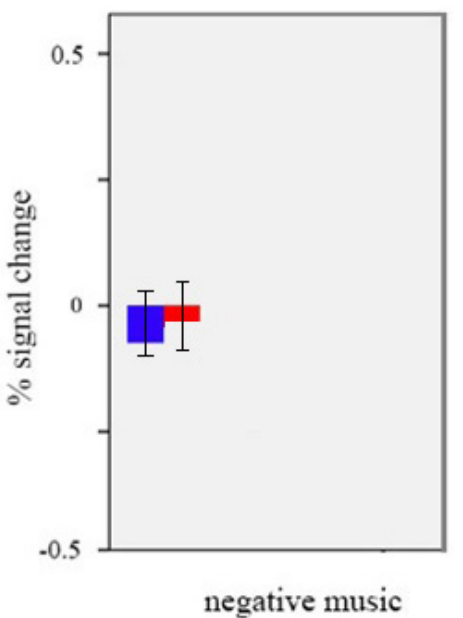

(b)

ACC showed differential task activation between ND and MDD groups to positive vs negative stimuli. Graphs show mean activation over the entire ROI. Error bars denote standard error. 
Gaussian likelihood function with uncorrelated errors in (3), and the posterior estimates are obtained.

The ND and MDD subjects' scans are processed for the contrast of positive music versus negative music stimuli in a run of musical stimuli. Posterior mean using model (3) for both ND and MDD subjects is computed. In the normal participants, Figure 3 shows the brain regions that were activated when subjected to the musical stimuli of contrast positive versus negative in the ND and MDD. Figure 3A displays the ACC mask, and the voxels of this region are shown in blue.

Within the ACC mask, there is a greater activation to positive versus negative music stimuli in ND and MDD groups. This activation for the ND group is higher than the MDD group (Figure $3 \mathrm{~B}-\mathrm{C}$ ). The white and yellow colors indicate the stronger activation, and the orange and red colors represent a weaker and zero activation, respectively.

The results show higher activation in emotional stimuli areas for the ND subjects than the MDD subjects. The results focused on the ACC showed a response to emotional stimuli in ND subjects. The ACC is highly activated due to an emotional musical stimulus. The entire ACC receives projections from the midbrain dopaminergic neurons and is implicated in emotional functioning among the ND subjects, whereas the MDD subjects lack the same.

Within ACC ROI and in comparison with MDD and ND individuals, a significant difference is observed between MDD and ND groups in response to positive music vs negative music stimuli. In ND, subject activations increase from baseline to positive stimuli and decrease from baseline to negative stimuli. In contrast, participants with depression showed no difference from baseline to negative stimuli and a significant decrease to positive stimuli (FigurE 4A-B).

\section{Discussion}

In this study, a recently-developed Bayesian inference approach based on INLA was employed. To the best of the authors' knowledge, this is the first research that applies the INLA algorithm for inferences of the described model in volumetric fMRI data. This Bayesian GLM approach was used in depressed subjects. Data downloaded from the OpenfMRI database. This experiment was designed to investigate the neural processing of emotion and reward in depression.
Activated a priori defined ACC was considered for analysis, and activation was compared within the anatomic mask and between two groups, one experiencing a current depressive (MDD) and ND group who has no history of depression or other psychiatric disorder. Both groups reported similar emotional experiences from the stimuli. In both ND and MDD groups, positive stimuli activated ACC to a greater amount than negative stimuli. Dopamine projections are received from the ventral tegmental area by the vACC and are sent dorsally and laterally to executive control areas of the cortex (Reeve, 2014). Blunted activation in this region has been associated with transient sadness (Devinsky, Morrell, \& Vogt, 1995) and with depression (Davidson, Pizzagalli, Nitschke, \& Putnam, 2002), suggesting that this region is critical for the experience of positive emotions. These findings confirm previous research (Menon \& Levitin, 2005; Salimpoor, Benovoy, Larcher, Dagher, \& Zatorre, 2011) and propose that during music listening, the dopaminergic system is active.

Over the last decade, many researchers have investigated the brain function in response to musical stimuli (Blood \& Zatorre, 2001; Levitin \& Tirovolas, 2009; Menon \& Levitin, 2005; Peretz \& Zatorre, 2005) and have indicated that music releases brain responses in reward centers (Menon \& Levitin, 2005; Salimpoor et al., 2011). One study explored how major depressive disorder affects the neural reactivity to music manifested, which in depression neural responsiveness for one's favorite music is modified (Osuch et al., 2009).

In MDD and ND groups, when positive stimuli were compared to negative stimuli, ACC showed relatively more activation to positive stimuli in ND participants with a significant decrease from baseline to negative stimuli. Participants with depression showed a significant decrease from baseline to positive stimuli but no difference from baseline for negative stimuli.

The most impressive stimulation site for brain stimulation in treatment-resistant depression is sgACC (Hamani et al., 2011). Although the activation found in this research expands beyond the sgACC, the entire ACC receives projections from the midbrain dopaminergic neurons and is involved in emotional functioning in depression cases (Drevets, 2000, 2001; Drevets, Price, \& Furey, 2008). The current findings from this fMRI data and the use of emotional auditory stimuli corroborate results found by Osuch et al. (2009). They showed that individuals with depression had reduced activation to music in this area. The pattern of activations within ACC in participants with depression makes the question of whether music and specifi- 
cally positive music, may be useful in retraining the ACC and improving functioning. Further research is required for confirming activation in ACC through a longitudinal study with a music intervention.

A new Bayesian GLM approach was proposed by Mejia et al. (2020) and was applied on cortical surface fMRI data from the Human Connectome Project. They mapped the volumetric fMRI data to the cortical surface manifold then used INLA for computational approximation (Mejia et al., 2020). In their study, the stochastic partial differential equation was considered for spatial correlation and entered the model via activation parameters. In the present study, the UGL prior was chosen for activation parameters. Also, one could apply another type of spatial prior such as intrinsic Conditional $\mathrm{Au}-$ toregressive (CAR) prior (Keefe, Ferreira, \& Franck, 2019). Most of the Bayesian methods for fMRI data reduce computational trouble using Variational Bayes (VB). However, VB underestimates posterior. In this study, the INLA approach was employed for inferences. INLA is a computationally efficient but highly accurate approximate Bayesian inference tool. Since INLA is less computationally demanding than MCMC, it made the researchers capable of fitting a complex model based on UGL spatial prior in order to consider spatial correlation of voxels appropriately.

In our work, since a predefined ACC area is considered, one can assess the whole brain in response to music stimuli for finding more corresponding regions. Also, single-subject analysis is conducted in the present research. One can perform multi-subject analysis using the Bayesian GLM approach proposed by Mejia et al. (2020). In a similar study, Hamiltonian Monte Carlo (HMC) algorithm was applied for Bayesian inferences in $\mathrm{fMRI}$ data. For comparison purposes, one could apply HMC or MCMC for statistical inferences (Sidén et al., 2017; Teng et al., 2017). Based on the literature, genetic factors influence emotion regulation (Hawn, Overstreet, Stewart, \& Amstadter, 2015). Accordingly, besides fMRI data, by collecting genetic information, more research could be done for assessing emotional and cognitive processes by modifying genetic factors.

\section{Conclusions}

The current study showed that in a healthy individual, positive auditory stimuli activated reward-processing areas of the brain that are involved in depression. Similarly, the activation pattern within ACC in a depressed individual may be useful and effective in retraining the ACC and improving functioning, leading to therapeutic interventions.

\section{Ethical Considerations}

\section{Compliance with ethical guidelines}

The fMRI data used in this study were downloaded from the OpenfMRI database. Its accession number is ds000171. This sentence was highlighted in the text (p. 4; 11. 29-31).

\section{Funding}

This article is a part of $\mathrm{PhD}$. dissertation of the first author and is supported by a grant from the Department of Biostatistics of Shahid Beheshti University of Medical Sciences, Tehran (Grant number: IR.SBMU.RETECH. REC.1397.602).

\section{Authors' contributions}

All authors equally contributed to preparing this article.

\section{Conflict of interest}

The authors declared no conflicts of interest.

\section{Acknowledgments}

This work was supported by a grant from the Department of Biostatistics of Shahid Beheshti University of Medical Sciences, Tehran, Iran.

\section{References}

Aselton, P. (2012). Sources of stress and coping in American college students who have been diagnosed with depression. Journal of Child and Adolescent Psychiatric Nursing, 25(3), 11923. [DOI:10.1111/j.1744-6171.2012.00341.x] [PMID]

Blangiardo, M., \& Cameletti, M. (2015). Spatial and spatio-temporal Bayesian models with R-INLA. Hoboken, New Jersey: John Wiley \& Sons. [DOI:10.1002/9781118950203]

Blood, A. J., \& Zatorre, R. J. (2001). Intensely pleasurable responses to music correlate with activity in brain regions implicated in reward and emotion. Proceedings of the National Academy of Sciences, 98(20), 11818-23. [DOI:10.1073/pnas.191355898] [PMID] [PMCID]

Bollmann, S., Puckett, A. M., Cunnington, R., \& Barth, M (2018). Serial correlations in single-subject fMRI with subsecond TR. NeuroImage, 166, 152-66. [DOI:10.1016/j.neuroimage.2017.10.043] [PMID]

Çelik, E., Dar, S. U. H., Yılmaz, Ö., Keleş, Ü., \& Çukur, T. (2019) Spatially informed voxelwise modeling for naturalistic fMRI experiments. NeuroImage, 186, 741-57. [DOI:10.1016/j.neuroimage.2018.11.044] [PMID] 
Clark, D. M. (1983). On the induction of depressed mood in the laboratory: Evaluation and comparison of the Velten and musical procedures. Advances in Behaviour Research and Therapy, 5(1), 27-49. [DOI:10.1016/0146-6402(83)90014-0]

Clark, D. M., \& Teasdale, J. D. (1985). Constraints on the effects of mood on memory. Journal of Personality and Social Psychology, 48(6), 1595-608. [DOI:10.1037/0022-3514.48.6.1595]

Daliri, M. R., \& Behroozi, M. (2012). fMRI: Clinical and research applications. OMICS Journal of Radiology, 1(4), e112. https:// www.researchgate.net/profile/Mehdi_Behroozi/publication/273360988

Davidson, R. J., Pizzagalli, D., Nitschke, J. B., \& Putnam, K. (2002). Depression: Perspectives from affective neuroscience. Annual Review of Psychology, 53(1), 545-74. [DOI:10.1146/annurev.psych.53.100901.135148] [PMID]

Devinsky, O., Morrell, M. J., \& Vogt, B. A. (1995). Contributions of anterior cingulate cortex to behaviour. Brain, 118(1), 279306. [DOI:10.1093/brain/118.1.279] [PMID]

Drevets, W. C. (2000). Functional anatomical abnormalities in limbic and prefrontal cortical structures in major depression. Progress in Brain Research, 126, 413-31. [DOI:10.1016/S00796123(00)26027-5]

Drevets, W. C. (2001). Neuroimaging and neuropathological studies of depression: implications for the cognitive-emotional features of mood disorders. Current Opinion in Neurobiology, 11(2), 240-9. [DOI:10.1016/S0959-4388(00)00203-8]

Drevets, W. C., Price, J. L., \& Furey, M. L. (2008). Brain structural and functional abnormalities in mood disorders: Implications for neurocircuitry models of depression. Brain Structure and Function, 213(1-2), 93-118. [DOI:10.1007/s00429-008-0189-x] [PMID] [PMCID]

Gross, J. J., \& Muñoz, R. F. (1995). Emotion regulation and mental health. Clinical psychology: Science and Practice, 2(2), 15164. [DOI:10.1111/j.1468-2850.1995.tb00036.x]

Hamani, C., Mayberg, H., Stone, S., Laxton, A., Haber, S., \& Lozano, A. M. (2011). The subcallosal cingulate gyrus in the context of major depression. Biological Psychiatry, 69(4), 301-8. [DOI:10.1016/j.biopsych.2010.09.034] [PMID]

Hawn, S. E., Overstreet, C., Stewart, K. E., \& Amstadter, A. B. (2015). Recent advances in the genetics of emotion regulation: A review. Current Opinion in Psychology, 3, 108-16. [DOI:10.1016/j.copsyc.2014.12.014] [PMID] [PMCID]

Holzinger, A., Matschinger, H., \& Angermeyer, M. (2012). What to do about depression? Self-help recommendations of the public. International Journal of Social Psychiatry, 58(4), 343-9. [DOI:10.1177/0020764010397262] [PMID]

Keefe, M. J., Ferreira, M. A., \& Franck, C. T. (2019). Objective Bayesian analysis for gaussian hierarchical models with intrinsic conditional autoregressive priors. Bayesian Analysis, 14(1), 181-209. [DOI:10.1214/18-BA1107]

Lepping, R. J., Atchley, R. A., Chrysikou, E., Martin, L. E., Clair A. A., \& Ingram, R. E., et al. (2016). Neural processing of emotional musical and nonmusical stimuli in depression. PloS One, 11(6), e0156859. [DOI:10.1371/journal.pone.0156859] [PMID] [PMCID]

Levitin, D. J., \& Tirovolas, A. K. (2009). Current advances in the cognitive neuroscience of music. Annals of the New York
Academy of Sciences, 1156(1), 211-31. [DOI:10.1111/j.17496632.2009.04417.x] [PMID]

Lindquist, M. A. (2008). The statistical analysis of fMRI data. Statistical Science, 23(4), 439-464. [DOI:10.1214/09-STS282]

Maldjian, J. A., Laurienti, P. J., Kraft, R. A., \& Burdette, J. H. (2003). An automated method for neuroanatomic and cytoarchitectonic atlas-based interrogation of fMRI data sets. NeuroImage, 19(3), 1233-9. [DOI:10.1016/S1053-8119(03)00169-1]

Mejia, A. F., Yue, Y., Bolin, D., Lindgren, F., \& Lindquist, M. A. (2020). A Bayesian general linear modeling approach to cortical surface fMRI data analysis. Journal of the American Statistical Association, 115(530), 501-20. https://www.tandfonline. com/doi/abs/10.1080/01621459.2019.1611582

Menon, V., \& Levitin, D. J. (2005). The rewards of music listening: Response and physiological connectivity of the mesolimbic system. NeuroImage, 28(1), 175-84. [DOI:10.1016/j.neuroimage.2005.05.053] [PMID]

Monti, M. M. (2011). Statistical analysis of fMRI time-series: a critical review of the GLM approach. Frontiers in Human Neuroscience, 5, 28. [DOI:10.3389/fnhum.2011.00028] [PMID] [PMCID]

Moussavi, S., Chatterji, S., Verdes, E., Tandon, A., Patel, V., \& Ustun, B. (2007). Depression, chronic diseases, and decrements in health: results from the World Health Surveys. The Lancet, 370(9590), 851-858. [DOI:10.1016/S0140-6736(07)61415-9]

Nazari, A., Alavimajd, H., Shakeri, N., Bakhshandeh, M., Faghihzadeh, E., \& Marzbani, H. (2019). Prediction of brain connectivity map in resting-state fmri data using shrinkage estimator. Basic and Clinical Neuroscience, 10(2), 147-56. [DOI:10.32598/ben.9.10.140] [PMID] [PMCID]

Oishi, K., Faria, A., Jiang, H., Li, X., Akhter, K., \& Zhang, J., et al. (2009). Atlas-based whole brain white matter analysis using large deformation diffeomorphic metric mapping: application to normal elderly and Alzheimer's disease participants. Neuroimage, 46(2), 486-99. [DOI:10.1016/j.neuroimage.2009.01.002] [PMID] [PMCID]

World Health Organization. (2000). The world health report 2000: health systems: Improving performance. Geneva: World Health Organization. https:/ /www.who.int/whr/2000/en/

Osuch, E. A., Bluhm, R. L., Williamson, P. C., Theberge, J., Densmore, M., \& Neufeld, R. W. (2009). Brain activation to favorite music in healthy controls and depressed patients. Neuroreport, 20(13), 1204-8. [DOI:10.1097/WNR.0b013e32832f4da3] [PMID]

Peretz, I., \& Zatorre, R. J. (2005). Brain organization for music processing. Annual Review of Psychology, 56, 89-114. [DOI:10.1146/annurev.psych.56.091103.070225] [PMID]

Pignatiello, M. F., Camp, C. J., \& Rasar, L. A. (1986). Musical mood induction: An alternative to the velten technique. Journal of Abnormal Psychology, 95(3), 295-9. [DOI:10.1037/0021843X.95.3.295] [PMID]

Poldrack, R. A., Mumford, J. A., \& Nichols, T. E. (2011). Handbook of functional MRI data analysis. Cambridge: Cambridge University Press. [DOI:10.1017/CBO9780511895029]

Raichle, M. E. (2003). Functional brain imaging and human brain function. Journal of Neuroscience, 23(10),3959-62. [DOI:10.1523/ JNEUROSCI.23-10-03959.2003] [PMID] [PMCID] 
Reeve, J. (2014). Understanding motivation and emotion. Hoboken: John Wiley \& Sons. https:/ / www.wiley.com/en-us/

Rue, H., Martino, S., \& Chopin, N. (2009). Approximate Bayesian inference for latent Gaussian models by using integrated nested Laplace approximations. Journal of the Royal Statistical Society: Series B (Statistical Methodology), 71(2), 319-92. [DOI:10.1111/j.1467-9868.2008.00700.x]

Rue, H., Riebler, A., Sørbye, S. H., Illian, J. B., Simpson, D. P., \& Lindgren, F. K. (2017). Bayesian computing with INLA: A review. Annual Review of Statistics and Its Application, 4, 395-421. [DOI:10.1146/annurev-statistics-060116-054045]

Salimpoor, V. N., Benovoy, M., Larcher, K., Dagher, A., \& Zatorre, R. J. (2011). Anatomically distinct dopamine release during anticipation and experience of peak emotion to music. Nature Neuroscience, 14(2), 257. [DOI:10.1038/nn.2726] [PMID]

Sidén, P., Eklund, A., Bolin, D., \& Villani, M. (2017). Fast Bayesian whole-brain $\mathrm{fMRI}$ analysis with spatial 3D priors. NeuroImage, 146, 211-25. [DOI:10.1016/j.neuroimage.2016.11.040] [PMID]

Sloboda, J. A., \& Juslin, P. N. (2001). Music and emotion: Theory and research. Oxford: Oxford University Press.

Sutherland, G., Newman, B., \& Rachman, S. (1982). Experimental investigations of the relations between mood and intrusive unwanted cognitions. Psychology and Psychotherapy: Theory, Research and Practice, 55(2), 127-38. [DOI:10.1111/j.2044-8341.1982.tb01491.x] [PMID]

Teng, M., Nathoo, F. S., \& Johnson, T. D. (2017). Bayesian Analysis of fMRI data with Spatially-Varying Autoregressive Orders. arXiv preprint arXiv:1710.01434. https://arxiv.org/ abs/1710.01434

Västfjäll, D. (2001). Emotion induction through music: A review of the musical mood induction procedure. Musicae Scientiae, 5(suppl 1), 173-211. [DOI:10.1177/10298649020050S107] 
This Page Intentionally Left Blank 